\title{
NORIYUKI SUWA
}

\section{TSUTOMU SEKIGUCHI}

\section{Théorie de Kummer-Artin-Schreier et applications}

Journal de Théorie des Nombres de Bordeaux, tome 7, no 1 (1995), p. $177-189$

<http://www.numdam.org/item?id=JTNB_1995_7_1_177_0>

(C) Université Bordeaux 1, 1995, tous droits réservés.

L'accès aux archives de la revue «Journal de Théorie des Nombres de Bordeaux » (http://jtnb.cedram.org/) implique l'accord avec les conditions générales d'utilisation (http://www.numdam.org/conditions). Toute utilisation commerciale ou impression systématique est constitutive d'une infraction pénale. Toute copie ou impression de ce fichier doit contenir la présente mention de copyright.

\section{Numdam}

Article numérisé dans le cadre du programme

Numérisation de documents anciens mathématiques

http://www.numdam.org/ 
Journal de Théorie des Nombres

de Bordeaux 7 (1995), 177-189

\title{
Théorie de Kummer-Artin-Schreier et applications
}

\author{
par Noriyuki SUwa et Tsutomu SeKIgUCHI
}

\section{Introduction}

Soit $p$ un nombre premier et soit $K$ un corps. La théorie de Kummer dit que si $K$ est de caractéristique $\neq p$ et $K$ contient les racines $p$-ième de l'unité, toute extension cyclique de $K$ de degré $p$ est obtenue par adjonction d'une racine $p$-ième d'un élément de $K$. Ceci est une conséquence des faits suivants:

(1) Si $K$ est de caractéristique $\neq p$, la suite de schémas en groupes sur $K$

$$
0 \rightarrow \mu_{p, K} \rightarrow \mathbb{G}_{m, K} \stackrel{p}{\rightarrow} \mathbb{G}_{m, K} \rightarrow 0
$$

est exacte pour la topologie étale sur $\operatorname{Spec} K\left(\mathbb{G}_{m, K}\right.$ est le groupe multiplicatif sur $K$ );

(2) (Hilbert 90) $\mathrm{H}_{\mathrm{et}}^{1}\left(K, \mathbb{G}_{m, K}\right)=0$.

D'autre part, la théorie d'Artin-Schreier dit que si $K$ est de caractéristique $p$, toute extension cyclique de $K$ de degré $p$ est obtenue par adjonction d'une racine de l'equation $T^{p}-T=a(a \in K)$. Ceci est une conséquence des faits suivants:

(1) Si $K$ est de caractéristique $p$, la suite de schémas en groupes sur $K$

$$
0 \rightarrow \mathbb{Z} / p \rightarrow \mathbb{G}_{a, K} \stackrel{F-1}{\longrightarrow} \mathbb{G}_{a, K} \rightarrow 0
$$

est exacte pour la topologie étale sur $\operatorname{Spec} K\left(\mathbb{G}_{a, K}\right.$ est le groupe additif sur $K$ );

(2) $\mathrm{H}_{\mathrm{et}}^{1}\left(K, \mathbb{G}_{a, K}\right)=0$.

(Voir SGA 4, Exp. IX pour un argument extrêmement développé.)

Il est intéressant de décrire les extensions cycliques non-ramifiées de degré $p$ d'un anneau $A$ de caractéristiques mixtes 0 et $p$. Quand $A$ contient les racines $p$-iémes de l'unité, ce problème a été résolu par Furtwängler (théorie des nombres $p$-primaires [1], [2]) bien qu'il ait restreint son étude

Manuscrit reçu le 5 Mars 1994. 
à l'anneau des entiers d'un corps de nombres. Récemment ses résultats ont été formulés dans le cadre de la théorie de cohomologie de schémas en groupes par Waterhouse [10] et [6] indépendamment. Dans cet article, nous expliquons brièvement l'esquisse de la théorie et ses applications.

Voici l'organisation de l'article un peu plus en détail. Nous rappelons quelques formalismes sur les schémas en groupes reliant le groupe multiplicatif $\mathbb{G}_{m}$ et le groupe additif $\mathbb{G}_{a}$ dans la section 1 , et la suite exacte de schémas en groupes unifiant les suites exactes de Kummer et d'ArtinSchreier. La plupart des résultats sont retirés de [6], qu'on consultera pour les détails. Nous ajoutons une description des extensions non-ramifiées cycliques d'ordre $p$ d'un anneau local, analogue aux théories de Kummer et d'Artin-Schreier rappelées au début dans l'introduction. Ce cas le plus élémentaire a échappé à [10] et à [6]. Dans la section 3 nous développons pour les schémas arithmétiques l'argument de Furtwängler [1], [2], surtout la théorie de nombres $p$-primaires. On arrive enfin à un acconplement nondégénéré $\mathrm{CH}_{0}(X) \times \mathcal{E}(X) \rightarrow \mu_{p}$ quand $X$ est régulier, plat et projectif sur l'anneau des entiers d'un corps de nombres contenant $\mathbb{Q}\left(\mu_{p}\right)$.

Nous tenons à remercier Humio Ichimura pour nous avoir introduit aux travaux de Furtwängler.

\section{Notations}

Pour un schéma $S$,

$\mathbb{G}_{a, S}:$ le groupe additif sur $S$

$\mathbb{G}_{m, S}:$ le groupe multiplicatif sur $S$

$\boldsymbol{\mu}_{p, S}=\operatorname{Ker}\left(p: \mathbb{G}_{m, S} \rightarrow \mathbb{G}_{m, S}\right)$

$\operatorname{Pic}(S)=\mathrm{H}^{1}\left(S, \mathbb{G}_{m, S}\right)$ : le groupe de Picard de $S$

Pour un anneau commutatif $B$,

$B^{\times}=\mathbb{G}_{m}(B):$ le groupe multiplicatif des unités de $B$

$\operatorname{Pic}(B)=\operatorname{Pic}(\operatorname{Spec} B):$ le group de Picard de $B$

$\mathrm{H}^{1}(B, \mathbb{Z} / p)=\mathrm{H}^{1}(\operatorname{Spec} B, \mathbb{Z} / p)$

Les groupes de cohomologie sont les groupes de cohomologie étale. 


\section{Schéma en groupes $\mathcal{G}^{(\lambda)}$}

\section{1 .}

Soient $A$ un anneau (commutatif), $\lambda \in A$ et $A_{0}=A /(\lambda)$. Définissons un schéma en groupes commutatifs $\mathcal{G}^{(\lambda)}$, lisse et affine sur $A$ par $\mathcal{G}^{(\lambda)}=$ $\operatorname{Spec} A\left[T, \frac{1}{\lambda T+1}\right]$ muni de loi de groupe:

(1) (multiplication) $T \mapsto \lambda T \otimes T+T \otimes 1+1 \otimes T$;

(2) (unité) $T \mapsto 0$;

(3) (inverse) $T \mapsto-T /(\lambda T+1)$.

D'ailleurs on définit un homomorphisme de schémas en groupes

$$
\alpha^{(\lambda)}: \mathcal{G}^{(\lambda)}=\operatorname{Spec} A\left[T, \frac{1}{\lambda T+1}\right] \rightarrow \mathbb{G}_{m, A}=\operatorname{Spec} A\left[U, \frac{1}{U}\right]
$$

par

$$
U \mapsto \lambda T+1: A\left[U, \frac{1}{U}\right] \rightarrow A\left[T, \frac{1}{\lambda T+1}\right] .
$$

Si $\lambda$ est inversible dans $A, \alpha^{(\lambda)}: \mathcal{G}^{(\lambda)} \rightarrow \mathbb{G}_{m, A}$ est un $A$-isomorphisme. D'autre part, si $\lambda=0, \mathcal{G}^{(\lambda)}$ n'est autre que le groupe additif $\mathbb{G}_{a, A}$. (cf. [6], Ch.I.) On remarquera que $\alpha^{(\lambda)}: \mathcal{G}^{(\lambda)} \rightarrow \mathbb{G}_{m, A}$ n'est pas injectif si $\lambda$ n'est pas inversible; $\operatorname{Ker}\left[\alpha^{(\lambda)}: \mathcal{G}^{(\lambda)} \rightarrow \mathbb{G}_{m, A}\right]$ est isomorphe au noyau de l'homothétie sur $\mathbb{G}_{a, A}$ par $\lambda$.

THÉORÈmE 1.2. Soit $X$ un A-schéma. Alors la suite de faisceaux abéliens sur $X$

$$
0 \rightarrow \mathbb{G}\{\lambda\} \rightarrow \mathcal{G}^{(\lambda)} \stackrel{\alpha^{(\lambda)}}{\longrightarrow} \mathbb{G}_{m, X} \rightarrow i_{*} \mathbb{G}_{m, X_{0}} \rightarrow 0
$$

est exacte pour la topologie étale. $\left(X_{0}=X \otimes_{A} A_{0}\right.$ et $i: X_{0} \rightarrow X$ désigne l'immersion fermée canonique, et $\mathbb{G}_{a}\{\lambda\}=\operatorname{Ker}\left[\lambda: \mathbb{G}_{a, X} \rightarrow \mathbb{G}_{a, X}\right]$.) Si $\lambda$ n'est pas diviseur de zéro et $X$ est plat sur $A$, la suite de faisceaux abéliens sur $X$

$$
0 \rightarrow \mathcal{G}^{(\lambda)} \stackrel{\alpha^{(\lambda)}}{\longrightarrow} \mathbb{G}_{m, X} \rightarrow i_{*} \mathbb{G}_{m, X_{0}} \rightarrow 0
$$

est exacte pour la topologie étale.

On peut vérifier l'assertion, en passant à la fibre géométrique en chaque point géométrique de $X$. (cf. [6], Ch.II, 1.1) 
Corollaire 1.3. (Hilbert 90) Si A est un anneau local, on a

$$
\mathrm{H}^{1}\left(A, \mathcal{G}^{(\lambda)}\right)=0
$$

En effet, posons $G=\operatorname{Ker}\left[\mathbb{G}_{m, A} \rightarrow i_{*} \mathbb{G}_{m, A}\right]$. Alors on obtient une suite exacte

$$
A^{\times} \rightarrow A_{0}^{\times} \rightarrow \mathrm{H}^{1}(A, G) \rightarrow \mathrm{H}^{1}\left(A, \mathbb{G}_{m, A}\right) .
$$

L'application canonique $A^{\times} \rightarrow A_{0}^{\times}$est surjective et $\mathrm{H}^{1}\left(A, \mathbb{G}_{m, A}\right)=\operatorname{Pic}(A)=$ $0, A$ étant local, et par suite $\mathrm{H}^{1}(A, G)=0$. Considérons maintenant une suite exacte

$$
\mathrm{H}^{1}\left(A, \mathbb{G}_{a}\{\lambda\}\right) \rightarrow \mathrm{H}^{1}\left(A, \mathcal{G}^{(\lambda)}\right) \rightarrow \mathrm{H}^{1}(A, G) .
$$

$\mathrm{H}^{1}\left(A, \mathbb{G}_{a}\{\lambda\}\right)=0$ comme $\mathbb{G}_{a}\{\lambda\}$ représente un faisceau quasi-cohérent sur $X_{\text {et }}$. D'où le résultat.

Remarque 1.4. Si $\lambda$ est nilpotent, on a

$$
\mathrm{H}^{1}\left(A, \mathcal{G}^{(\lambda)}\right)=0
$$

même si $A$ n'est pas local.

\section{5 .}

Soient $X$ un schéma et $Y$ un sous-schéma fermé de $X$. Soient $\mathcal{L}, \mathcal{L}^{\prime}$ des faisceaux inversibles sur $X$, triviaux sur $Y$ et $h: \mathcal{O}_{Y} \stackrel{\sim}{\rightarrow} \mathcal{O}_{Y} \otimes_{\mathcal{O}_{X}} \mathcal{L}$,

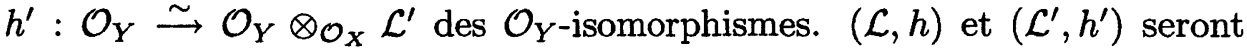
dit équivalents s'il existe un $\mathcal{O}_{X}$-isomorphisme $g: \mathcal{L} \stackrel{\sim}{\rightarrow} \mathcal{L}^{\prime}$ tel que le diagramme

$$
\mathcal{O}_{Y} \otimes_{\mathcal{O}_{X}} \mathcal{L}^{h \swarrow} \underset{1 \otimes g}{\stackrel{\mathcal{O}_{Y}}{\longrightarrow}} \searrow_{h^{\prime}} \mathcal{O}_{Y} \otimes_{\mathcal{O}_{X}} \mathcal{L}^{\prime}
$$

siot commutatif. Posons

$$
\operatorname{Pic}(X, Y)=\left\{\begin{array}{l|l}
(\mathcal{L}, h) & \begin{array}{l}
\mathcal{L} \text { est un faisceau invertible sur } X, \text { triv- } \\
\text { ial sur } Y, h: \mathcal{O}_{Y} \stackrel{\sim}{\rightarrow} \mathcal{O}_{Y} \otimes_{\mathcal{O}_{X}} \mathcal{L} \text { est un } \\
\mathcal{O}_{Y} \text {-isomorphisme }
\end{array}
\end{array} / \sim\right.
$$

Définissons une multiplication sur $\operatorname{Pic}(X, Y) \operatorname{par}(\mathcal{L}, h) \cdot\left(\mathcal{L}^{\prime}, h^{\prime}\right)=\left(\mathcal{L} \otimes_{\mathcal{O}_{X}}\right.$ $\left.\mathcal{L}^{\prime}, h h^{\prime}\right)$. Alors $\operatorname{Pic}(X, Y)$ est un groupe commutatif. Si $Y$ est vide, $\operatorname{Pic}(X, Y)$ n'est autre que le groupe de Picard de $X$.

On suppose maintenant que $\lambda$ n'est pas diviseur de zéro et $X$ est plat sur $A$. Soit $X_{0}=X \otimes_{A} A_{0}$. Alors $\mathrm{H}^{1}\left(X, \mathcal{G}^{(\lambda)}\right)$ est isomorphe à $\operatorname{Pic}\left(X, X_{0}\right)$. 
EXemple 1.6. ([6], Ch.II, 1.5 et 2.9) Soit $f: X \rightarrow \operatorname{Spec} A$ un morphisme propre et plat, à fibres connexes et réduites sur $A$. Si $\lambda$ n'est pas diviseur de zéro, on a

$$
\mathrm{R}^{1} f_{*} \mathcal{G}^{(\lambda)}=\operatorname{Ker}\left[\underline{\mathrm{Pic}}_{X / A} \rightarrow i_{*} \underline{\mathrm{Pic}}_{X_{0} / A_{0}}\right] .
$$

De plus, si l'application canonique $A^{\times} \rightarrow A_{0}^{\times}$est surjective, on a

$$
\mathrm{H}^{1}\left(X, \mathcal{G}^{(\lambda)}\right)=\operatorname{Pic}\left(X, X_{0}\right)=\operatorname{Ker}\left[\operatorname{Pic}(X) \rightarrow \operatorname{Pic}\left(X_{0}\right)\right] .
$$

En particulier, si $A$ est un anneau local strictement hénselien et $X$ est un schéma abélien, on a

$$
\operatorname{Ext}^{1}\left(X, \mathcal{G}^{(\lambda)}\right)=\mathrm{H}^{1}\left(X, \mathcal{G}^{(\lambda)}\right)=\operatorname{Ker}\left[\operatorname{Pic}^{\circ}(X) \rightarrow \operatorname{Pic}^{\circ}\left(X_{0}\right)\right] .
$$

Reamrque 1.7.. Soient $X$ un schéma et $D$ un diviseur de Cartier effectif de $X$. Il existe alors un schéma en groupes commutatifs $\mathbb{G}_{m}(-D)$, lisse et affine sur $X$, tel que $\mathbb{G}_{m}(-D)$ soit isomorphe à $\operatorname{Ker}\left[\mathbb{G}_{m, X} \rightarrow i_{*} \mathbb{G}_{m, D}\right]$ sur le site étale de $X$. ( $i: D \rightarrow X$ désigne l'immersion fermée canonique.) D'ailleurs, $\mathrm{H}^{1}\left(X, \mathbb{G}_{m}(-D)\right)$ est isomorphe à $\mathrm{Pic}(X, D)$. Par exemple, si $A$ est l'anneau des entiers d'un corps de nombres $K, X=\operatorname{Spec} A$ et $D$ est un diviseur effectif de $X, \mathrm{H}^{1}\left(X, \mathbb{G}_{m}(-D)\right)=\operatorname{Pic}(X, D)$ est isomorphe au groupe des classes de rayon de $K$ modulo $D$.

\section{Théorie de Kummer-Artin-Schreier}

\section{1.}

Soit $p$ un nombre premier. Posons $\zeta=e^{2 \pi i / p}, \lambda=\zeta-1, A=\mathbb{Z}[\zeta]$ et $K=\mathbb{Q}(\zeta)$. Alors $(\lambda)$ est un idéal premier de $A$, et on a $(\lambda)^{p-1}=(p)$ dans $A$ et $A /(\lambda)=\mathbb{F}_{p}$.

Définissons un homomorphisme

$$
\Psi_{p}: \mathcal{G}^{(\lambda)}=\operatorname{Spec} A\left[T, \frac{1}{\lambda T+1}\right] \rightarrow \mathcal{G}^{\left(\lambda^{p}\right)}=\operatorname{Spec} A\left[T, \frac{1}{\lambda^{p} T+1}\right]
$$

de schémas en groupes sur $A$ par

$$
T \mapsto \frac{(\lambda T+1)^{p}-1}{\lambda^{p}}: A\left[T, \frac{1}{\lambda^{p} T+1}\right] \rightarrow A\left[T, \frac{1}{\lambda T+1}\right]
$$

Alors $\operatorname{Ker} \Psi_{p}=\operatorname{Spec} A\left[T, \frac{1}{\lambda T+1}\right] /\left(\frac{(\lambda T+1)^{p}-1}{\lambda^{p}}\right)$ est isomorphe au groupe constant $\mathbb{Z} / p$. D'ailleurs on a un diagramme commutatif de schémas 
en groupes sur $A$, avec lignes exactes

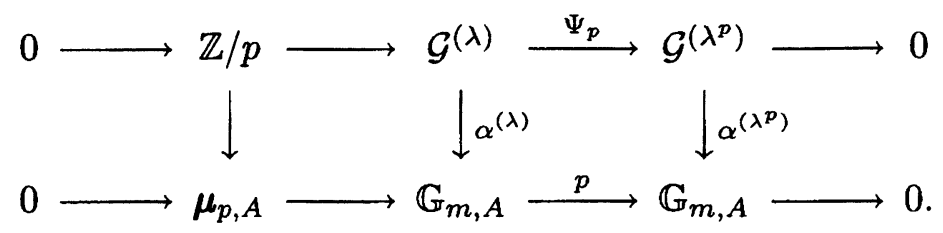

Par suite, la suite exacte

$$
\left[0 \rightarrow \mathbb{Z} / p \rightarrow \mathcal{G}^{(\lambda)} \stackrel{\Psi_{p}}{\longrightarrow} \mathcal{G}^{\left(\lambda^{p}\right)} \rightarrow 0\right] \otimes_{A} K
$$

est isomorphe à la suite de Kummer

$$
0 \rightarrow \mu_{p, K} \rightarrow \mathbb{G}_{m, K} \stackrel{p}{\rightarrow} \mathbb{G}_{m, K} \rightarrow 0 .
$$

D'autre part, la suite exacte

$$
\left[0 \rightarrow \mathbb{Z} / p \rightarrow \mathcal{G}^{(\lambda)} \stackrel{\Psi_{p}}{\longrightarrow} \mathcal{G}^{\left(\lambda^{p}\right)} \rightarrow 0\right] \otimes_{A} \mathbb{F}_{p}
$$

n'est autre que la suite d'Artin-Schreier

$$
0 \rightarrow \mathbb{Z} / p \rightarrow \mathbb{G}_{a, \mathbb{F}_{p}} \stackrel{F-1}{\longrightarrow} \mathbb{G}_{a, \mathbb{F}_{p}} \rightarrow 0 .
$$

On appellera la suite exacte

$$
0 \rightarrow \mathbb{Z} / p \rightarrow \mathcal{G}^{(\lambda)} \stackrel{\Psi_{p}}{\longrightarrow} \mathcal{G}^{\left(\lambda^{p}\right)} \rightarrow 0
$$

suite de Kummer-Artin-Schreier ou de Fourwängler (cf. [10], [6], Ch.II.).

Soient $X$ un $A$-schéma et $X_{0}=X \otimes_{A} \mathbb{F}_{p}$. En prenant la cohomologie, on obtient un diagramme commutatif au signe près, avec lignes exactes

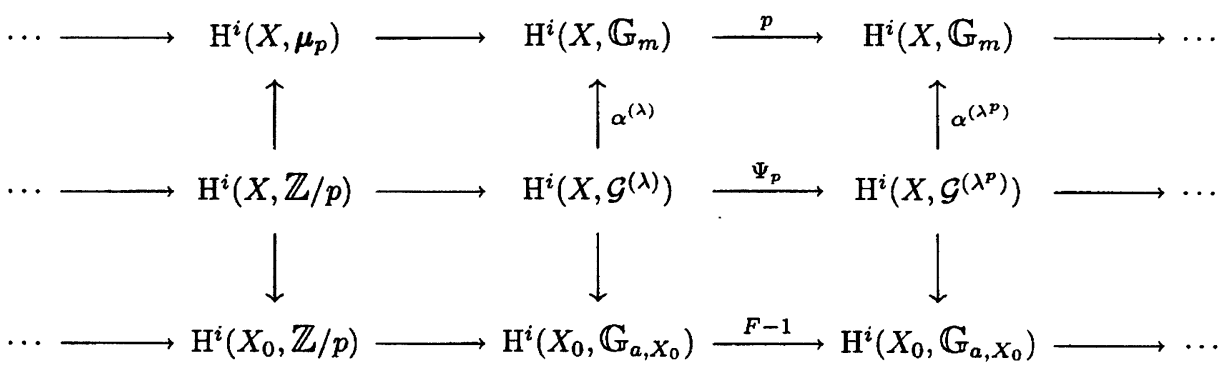

D'aprés 1.3 et 1.4 , on a le résultat suivant: 
ThÉORÈme 2.2. Soit $B$ une A-algèbre. On suppose que:

(1) $\lambda$ est nilpotent dans $B$, ou

(2) $B$ est un anneau local.

Alors $\mathrm{H}^{1}(B, \mathbb{Z} / p)$ est isomorphe à $\operatorname{Coker}\left[\Psi_{p}: \mathcal{G}^{(\lambda)}(B) \rightarrow \mathcal{G}^{\left(\lambda^{p}\right)}(B)\right]$.

\section{3.}

On peut formuler plus explicitement l'assertion de 2.2. Soit $B$ une $A$ algèbre locale. Alors toute extension de $B$, non-ramifiée cyclique de degré $p$ est obtenue par adjonction d'une racine de l'équation $\Psi_{p}(T)=a(a \in B)$. Soient

$$
C=B[\theta]=B[T] /\left(\Psi_{p}(T)-a\right)
$$

et

$$
\theta=T \bmod \Psi_{p}(T)-a .
$$

Alors $\operatorname{Gal}(C / B)=\mathbb{Z} / p$ est engendré par $\theta \mapsto \zeta \theta+1$. (Théorie de KummerArtin-Schreier). Posons d'ailleurs

$$
\Xi(T)=\frac{1}{p} \sum_{i=0}^{p-1}(\lambda T+1)^{i} \in \mathbb{Z}[\zeta][T] .
$$

Alors $\Xi(\Theta)$ engendre une base normale de l'extension $C / B$ non-ramifiée cyclique de degré $p$ (cf. [3], [7]).

\section{4.}

Soient maintenant $B$ une $A$-algèbre, $B_{0}=B /(\lambda)$ et $B_{1}=B /\left(\lambda^{p}\right)$. Posons

$$
\mathcal{E}_{2}(B)=\operatorname{Coker}\left[\Psi_{p}: \mathcal{G}^{(\lambda)}(B) \rightarrow \mathcal{G}^{\left(\lambda^{p}\right)}(B)\right]
$$

Si $B$ est plate sur $A$, on a une suite exacte

$$
\begin{aligned}
0 \rightarrow \mathcal{E}_{2}(B) & \rightarrow \mathrm{H}^{1}(B, \mathbb{Z} / p) \\
& \rightarrow \operatorname{Ker}\left[p: \operatorname{Pic}\left(B, B_{0}\right) \rightarrow \operatorname{Pic}\left(B, B_{1}\right)\right] \rightarrow 0
\end{aligned}
$$

Les éléments de $\mathcal{E}_{2}(B)$ correspondent aux extensions de $B$ non-ramifiée cycliques de degré $p$, admettant une base normale sur $B$. (cf. Childs [3]). 


\section{5.}

(cf.[6],Ch.II,3.2 et 3.4) En outre, soient $X$ un schéma propre et plat, à fibres connexes et réduites sur $B, X_{0}=X \otimes_{B} B_{0}$ et $X_{1}=X \otimes_{B} B_{1}$. Alors on a une suite exacte

$$
\begin{aligned}
0 \rightarrow \mathcal{E}_{2}(B) & \rightarrow \mathrm{H}^{1}(X, \mathbb{Z} / p) \\
& \rightarrow \operatorname{Ker}\left[p: \operatorname{Pic}\left(X, X_{0}\right) \rightarrow \operatorname{Pic}\left(X, X_{1}\right)\right] \rightarrow 0 .
\end{aligned}
$$

Si $B$ est un anneau local, on a

$$
\mathcal{E}_{2}(B)=\mathrm{H}^{1}(B, \mathbb{Z} / p)
$$

et

$\operatorname{Ker}\left[p: \operatorname{Pic}\left(X, X_{0}\right) \rightarrow \operatorname{Pic}\left(X, X_{1}\right)\right]=\operatorname{Ker}\left[{ }_{p} \operatorname{Pic}(X) \rightarrow{ }_{p} \operatorname{Pic}\left(X_{0}\right)\right]$ d'après 2.2 et 1.6. On en obtient une suite exacte

$$
\begin{aligned}
0 \rightarrow \mathrm{H}^{1}(B, \mathbb{Z} / p) & \rightarrow \mathrm{H}^{1}(X, \mathbb{Z} / p) \\
& \rightarrow \operatorname{Ker}\left[{ }_{p} \operatorname{Pic}(X) \rightarrow{ }_{p} \operatorname{Pic}\left(X_{0}\right)\right] \rightarrow 0 .
\end{aligned}
$$

En particulier, si $B$ est un anneau local strictement hénselien, on a

$$
\mathrm{H}^{1}(X, \mathbb{Z} / p)=\operatorname{Ker}\left[{ }_{p} \operatorname{Pic}(X) \rightarrow{ }_{p} \operatorname{Pic}\left(X_{0}\right)\right] .
$$

De plus, si $X$ est un schéma abélien sur $B$, on a

$$
\operatorname{Ext}_{A}^{1}(X, \mathbb{Z} / p)=\mathrm{H}^{1}(X, \mathbb{Z} / p)=\operatorname{Ker}\left[{ }_{p} \operatorname{Pic}^{0}(X) \rightarrow{ }_{p} \operatorname{Pic}^{0}\left(X_{0}\right)\right]
$$

\section{Autour de Furtwängler}

3.1.

Posons $\zeta=e^{2 \pi i / p}$ et $\lambda=\zeta-1$. Soient $L$ une extension algébrique de $\mathbb{Q}(\zeta), B$ l'anneau des entiers de $L$ et $B_{0}=B /(\lambda)$. Soient $X$ un schéma irréductible et réduit, plat sur $B$ et $K(X)$ le corps des fonctions rationnelles de $X$. Désignons par $\mathcal{O}_{Q}$ le localisé de $X$ en $Q$ et par $k(Q)$ le corps résiduel de $\mathcal{O}_{Q}$ pour chaque $Q \in X$. Soient $R: \mathrm{H}^{1}(X, \mathbb{Z} / p) \rightarrow \mathrm{H}^{1}(K(X), \mathbb{Z} / p)$ et $R_{Q}: \mathrm{H}^{1}\left(\mathcal{O}_{Q}, \mathbb{Z} / p\right) \rightarrow \mathrm{H}^{1}(K(X), \mathbb{Z} / p)$ les homomorphismes induits par les morphismes canoniques $\operatorname{Spec} K(X) \rightarrow X$ et $\operatorname{Spec} K(X) \rightarrow \operatorname{Spec}_{Q}$ réspectivement. Alors on a

$$
\operatorname{Im} R=\bigcap_{Q \in X} \operatorname{Im} R_{Q}
$$

D'après les théories de Kummer et de Kummer-Artin-Schreier, on a le résultat suivant: 
ThÉORÈme 3.2. Soit $X$ un schéma irréductible et réduit, plat sur B. Alors on $a$

$$
\begin{aligned}
\operatorname{Im}\left[R_{Q}: \mathrm{H}^{1}\left(\mathcal{O}_{Q}, \mathbb{Z} / p\right)\right. & \left.\rightarrow \mathrm{H}^{1}(K(X), \mathbb{Z} / p)=K(X)^{\times} /\left(K(X)^{\times}\right)^{p}\right] \\
& = \begin{cases}\mathcal{O}_{Q}^{\times} \cdot\left(K(X)^{\times}\right)^{p} /\left(K(X)^{\times}\right)^{p} & \text { ch. } k(Q) \neq p \\
\left(1+\lambda^{p} \mathcal{O}_{Q} \cdot\left(K(X)^{\times}\right)^{p} /\left(K(X)^{\times}\right)^{p}\right. & \text { ch. } k(Q)=p .\end{cases}
\end{aligned}
$$

\section{3.}

Soit $f \in K(X)^{\times}$. Nous dirons, en suivant Furtwängler ([1], Def. 10), que $f$ est p-primaire ou primaire (relativement à $X)$ si $f \in\left(1+\lambda^{p} \mathcal{O}_{Q}^{\times}\right)$. $\left(K(X)^{\times}\right)^{p}$ pour chaque point $Q \in X$ de craractéristique $p$.

ThÉORÈme 3.4. Soit $X$ un schéma connexe et normal, plat sur B. Alors on $a$

$\mathrm{H}^{1}(X, \mathbb{Z} / p)=\left\{\begin{array}{l|l}f \in K(X)^{\times} & \begin{array}{l}f \text { est primaire, } f \in \mathcal{O}_{Q}^{\times} \cdot\left(K(X)^{\times}\right)^{p} \text { pour } \\ \text { chaque } Q \in X\end{array}\end{array}\right\} /\left(K(X)^{\times}\right)^{p}$.

En effet, $X$ étant normal, $R: \mathrm{H}^{1}(X, \mathbb{Z} / p) \rightarrow \mathrm{H}^{i}(K(X), \mathbb{Z} / p)$ et $R_{Q}$ : $\mathrm{H}^{1}\left(\mathcal{O}_{Q}, \mathbb{Z} / p\right) \rightarrow \mathrm{H}^{1}(K(X), \mathbb{Z} / p)$ sont injectifs pour tout $Q \in X$ (SGA 1 , Exp.V, Prop.8.2.).

\section{5.}

Supposons maintenant $X$ régulier. Par le théorème de pureté de ZariskiNagata (SGA 2, Exp.X, Th.3.4.), on a

$$
\operatorname{Im} R=\bigcap_{\operatorname{codim} Q=1} \operatorname{Im} R_{Q}
$$

Soit $f \in K(X)^{\times}$. Soit $Q$ un point de $X$ de codimension 1. Alors $\mathcal{O}_{Q}$ est un anneau de valuation discrète. Désignons par $\operatorname{ord}_{Q}$ la valuation additive normalisée de $\mathcal{O}_{Q}$. Si $f \in \operatorname{Im} R_{Q}$, on a $\operatorname{ord}_{Q} f \equiv 0 \bmod p$ par 3.2. Donc, si $f \in \operatorname{Im} R_{Q}$ pour chaque $Q \in X$ de codimension 1 , il existe un diviseur $D$ de $X$ tel que $\operatorname{div}(f)=p D$.

Soit $f \in K(X)^{\times}$. Nous dirons, en suivant encore Furtwängler([2]), que $f$ est $p$-singulier ou singulier (relativement à $X$ ) s'il existe un diviseur $D$ de $X$ tel que $\operatorname{div}(f)=p D$. Posons

$$
\mathcal{E}(X)=\left\{f \in K(X)^{\times} \mid f \text { est singulier et primaire }\right\} /\left(K(X)^{\times}\right)^{p}
$$


et

$\mathcal{E}_{1}(X)=\left\{\begin{array}{l|l}f \in K(X)^{\times} & \begin{array}{l}f \text { est singulier et primaire, ord } Q f=0 \\ \text { pour tout point } Q \text { de } X \text { de codimension } \\ 1\end{array}\end{array}\right\} \cdot\left(K(X)^{\times}\right)^{p} /\left(K(X)^{\times}\right)^{p}$.

On obtient le résultat suivant:

ThÉORÈme 3.6. Soit $X$ un schéma connexe et régulier, plat sur $B$. Alors on $a$

$$
\mathrm{H}^{1}(X, \mathbb{Z} / p)=\mathcal{E}(X)
$$

et une suite exacte

$$
0 \rightarrow \mathcal{E}_{1}(X) \rightarrow \mathrm{H}^{1}(X, \mathbb{Z} / p) \rightarrow \operatorname{Ker}\left[{ }_{p} \operatorname{Pic}(X) \rightarrow{ }_{p} \operatorname{Pic}\left(X_{0}\right)\right]
$$

\section{7.}

On suppose maintnant que $L$ est fini sur $\mathbb{Q}(\zeta)$, et totalement imaginaire si $p=2$, et que $X$ est projectif sur $B$. Alors le groupe de Chow $\mathrm{CH}_{0}(X)$ des 0-cycles sur $X$ est fini et l'application d'Artin induit un isomorphisme $\mathrm{CH}_{0}(X) \stackrel{\sim}{\longrightarrow} \pi_{1}^{\mathrm{ab}}(X)$, compatible à l'action de $\operatorname{Gal}(L / \mathbb{Q})$ (Kato-Saito [4], Th.6.1 et Th.9.10). On a donc un $\operatorname{Gal}(L / \mathbb{Q})$-isomorphisme

$$
\operatorname{Hom}\left(\mathrm{CH}_{0}(X), \mathbb{Z} / p\right) \stackrel{\sim}{\longrightarrow} \mathrm{H}^{1}(X, \mathbb{Z} / p)
$$

comme $\mathrm{H}^{1}(X, \mathbb{Z} / p)=\operatorname{Hom}\left(\pi_{1}^{\mathrm{a} b}(X), \mathbb{Z} / p\right)$. D'autre part, l'injection

$$
\mathrm{H}^{1}(X, \mathbb{Z} / p) \rightarrow \mathrm{H}^{1}(K(X), \mathbb{Z} / p)=\mathrm{H}^{1}\left(K(X), \boldsymbol{\mu}_{p}\right) \otimes_{\mathbb{Z} / p} \boldsymbol{\mu}_{p}^{\otimes(-1)}
$$

induit un $\operatorname{Gal}(L / \mathbb{Q})$-isomorphisme

$$
\mathrm{H}^{1}(X, \mathbb{Z} / p) \stackrel{\sim}{\longrightarrow} \mathcal{E}(X) \otimes_{\mathbb{Z} / p} \boldsymbol{\mu}_{p}^{\otimes(-1)}
$$

On en obtient un $\operatorname{Gal}(L / \mathbb{Q})$-isomorphisme

$$
\mathcal{E}(X) \stackrel{\operatorname{Hom}}{ }\left(\mathrm{CH}_{0}(X), \mu_{p}\right)
$$

et une suite exacte de $\operatorname{Gal}(L / \mathbb{Q})$-modules

$$
0 \rightarrow \mathcal{E}_{1}(X) \rightarrow \operatorname{Hom}\left(\mathrm{CH}_{0}(X), \mu_{p}\right) \rightarrow \operatorname{Ker}\left[{ }_{p} \operatorname{Pic}(X) \rightarrow{ }_{p} \operatorname{Pic}\left(X_{0}\right)\right]
$$


Si les fibres de $X$ sur $B$ sont connexes et réduites, $\mathcal{E}_{1}(X)=\mathcal{E}_{1}(B)$. Par consé-quent, on obtient

$$
\operatorname{dim}_{\mathbb{F}_{p} p} \mathrm{CH}_{0}(X) \leq \operatorname{dim}_{\mathbb{F}_{p}} \mathcal{E}_{1}(B)+\operatorname{dim}_{\mathbb{F}_{p}} \operatorname{Ker}\left[{ }_{p} \operatorname{Pic}(X) \rightarrow{ }_{p} \operatorname{Pic}\left(X_{0}\right)\right] .
$$

De plus, si $\operatorname{Gal}(L / \mathbb{Q})$ est d'ordre premier à $p$, on a

$$
\begin{aligned}
\operatorname{dim}_{\mathbb{F}_{p} p} \mathrm{CH}_{0}(X)^{\left(\omega \chi^{-1}\right)} & \leq \operatorname{dim}_{\mathrm{F}_{p}} \mathcal{E}_{1}(B)^{(\chi)} \\
& +\operatorname{dim}_{\mathbb{F}_{p}} \operatorname{Ker}\left[{ }_{p} \operatorname{Pic}(X) \rightarrow{ }_{p} \operatorname{Pic}\left(X_{0}\right)\right]^{(\chi)}
\end{aligned}
$$

pour tout caractère $\chi: \operatorname{Gal}(L / \mathbb{Q}) \rightarrow \mathbb{F}_{p}^{\times}$. ( $\omega$ est le caractère cyclotomique et $E^{(\chi)}$ désigne la composante propre d'un $\mathbb{F}_{p}[\operatorname{Gal}(L / \mathbb{Q})]$-module $E$ pour un caractére $\chi: \operatorname{Gal}(L / \mathbb{Q}) \rightarrow \mathbb{F}_{p}^{\times}$.) Quand $X=\operatorname{Spec} B, \mathrm{CH}_{0}(X)=\operatorname{Pic}(X)$ est le groupe des classes d'idéaux de $L$, et on retrouve l'argument de Leopoldt sur le Spiegelungsatz [7].

\section{8 .}

Nous allons terminer cet article en donnant une déscription de l'isomorphisme $\mathcal{E}(X) \stackrel{\sim}{\rightarrow} \operatorname{Hom}\left(\mathrm{CH}_{0}(X), \mu_{p}\right)$.

Soient $f \in K(X)^{\times}$et $P$ un point fermé de $X$ de caractéristique $\neq p$. Quand $f \in \mathcal{O}_{P}$, on désigne par $\left(\frac{f}{P}\right)_{p}$ la racine $p$-ième telle que

$$
\left(\frac{f}{P}\right)_{p}=f^{(N(P)-1) / p} \text { dans } k(P),
$$

où $N(P)$ est l'ordre du corps résiduel $k(P)$.

Soit maintenant $Z=\sum_{P} n_{P} P$ un 0 -cycle de $X$, premier à $p$. Quand $f \in \bigcap_{\substack{P \\ n_{P} \neq 0}} \mathcal{O}_{P}$, on pose

$$
\left(\frac{f}{Z}\right)_{p}=\prod_{P}\left(\frac{f}{P}\right)_{p}^{n_{P}} .
$$

Par ailleurs, on voit que le symbole $\left(\frac{f}{Z}\right)_{p}$ est bien défini pour $f \in \mathcal{E}(X)$ et $Z \in \mathrm{CH}_{0}(X)$, en remarquant les faits suivants:

(1) si $f=g^{p}, g \in K(X)$, on a $\left(\frac{f}{Z}\right)_{p}=1$;

(2) si $f$ est singulier et primaire, et $Z$ est rationnellement équivalent à 0, on $\mathrm{a}\left(\frac{f}{Z}\right)_{p}=1$ 
La bijectivité de l'application d'Artin $\mathrm{CH}_{0}(X) \rightarrow \pi_{1}(X)^{\mathrm{ab}}$ entraîne que la forme bilinéaire

$$
(f, Z) \mapsto\left(\frac{f}{Z}\right)_{p}: \mathcal{E}(X) \times \mathrm{CH}_{0}(X) \rightarrow \mu_{p}
$$

est non-dégénérée.

Remarque 3.9. Il existe une suite exacte de schémas en groupes sur $\mathbb{Z}_{(p)}\left[\sqrt[p^{n}]{1}\right]$, qui unifie les suites de Kummer et d'Artin-Schreier-Witt de degré $p^{n}$. On en obtient une déscription des extensions non-ramifiées cycliques de degré $p^{n}$ d'un anneau de caractéristiques mixtes 0 et $p$. (cf. [8]. On consultera [9] pour les details.)

\section{REFERENCES}

[1] P. Furtwängler, Über die Reziprozitätsgesetze der $\ell$-ten Potenzreste in algebraischen Zahl-körpern, wenn $\ell$ eine ungerage Primzahl bedeutet, Math. Ann. 58 (1904), 1-50.

[2] - Allgemeiner Existenzbeweis für den Klassenkörper eines bebliegen Zahlkölpers, Math. Ann. 63 (1907), 1-37.

[3] L. N. Childs, The group of unramified Kummer extensions of prime degree, Proc. London Math. Soc. 35 (1977), 407-422.

[4] K. Kato and S. Saito, Global class field theory of arithmetic schemes, Contemporary Math. 55 (1986), 255-331.

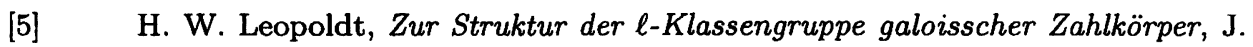
Reine Angew. Math. 199 (1958), 165-174.

[6] T. Sekiguchi, F. Oort and N. Suwa, On the deformation of Artin-Schreier to Kummer, Ann. Scient. Ec. Normale Sup. 22 (1989), 345-375.

[7] T. Sekiguchi and N. Suwa, Théorie de Kummer-Artin-Schreier, C. R. Acad. Sci. Paris 312 (1991), 417-420.

[8] , Théories de Kummer-Artin-Schreier-Witt, C. R. Acad. Sci. Paris 319 (1994), 105-110.

[9] - Unified Kummer-Artin-Schreier-Witt sequences, (en préparation).

[10] W. C. Waterhouse, A unified Kummer-Artin-Schreier sequence, Math. Ann. 277 (1987), 447-451.

[SGA 1] A. Grothendieck, Revêtements étales et groupe fondamental, Lecture Notes in Math 224 (1971).

[SGA 2] - Cohomologie local des faisceaux cohérents et théorèmes de Lefschetz locaux et globaux (1968), Masson, North-Holland.

[SGA 4] M. Artin, A. Grothendieck and J.-L. Verdier, Théorie des topos et cohomologie étale de schémas, Lecture Notes in Math. 269, 270, 305 (1972/73). 
Tsutomu SekiguchI

Department of Mathematics

Faculty of Science and Engineering

Chuo University

Kasuga 1-13-27, Bunkyo-ku

Tokyo 112, Japan

e-mail: sekiguti@math.chuo-u.ac.jp

Noriyuki Suwa

Department of Mathematics

Tokyo Deniki University

Kanda-Nisiki-cho 2-2, Chiyoda-ku

Tokyo 101, Japan

e-mail: suwa@cck.dendai.ac.jp 